\title{
Topological Bihyperbolic Modules
}

\author{
Merve Bilgin ${ }^{1}$, Soley Ersoy ${ }^{2 *}$
}

\section{Abstract}

The aim of this article is introducing and researching hyperbolic modules, bihyperbolic modules, topological hyperbolic modules and topological bihyperbolic modules. In this regard, we define balanced, convex and absorbing sets in hyperbolic and bihyperbolic modules. In particular, we investigate convex sets in hyperbolic numbers set (it is a hyperbolic module over itself) by considering the isomorphic relation of this set with 2-dimensional Minkowski space. Moreover, bihyperbolic numbers set is a bihyperbolic module over itself, too. So, we define convex sets in this module by considering hypersurfaces of 4-dimensional semi Euclidean space that are isomorphic to some subsets of bihyperbolic numbers set. We also study the interior and closure of some special sets and neighbourhoods of the unit element of the module in the introduced topological bihyperbolic modules. In the light of obtained results, new relationships are presented for idempotent representations in topological bihyperbolic modules.

Keywords: Bihyperbolic numbers, hyperbolic numbers, topological bihyperbolic modules, topological modules 2010 AMS: Primary 57N17, 52A07; Secondary 54B20

${ }_{1}^{1}$ Department of Mathematics, Faculty of Science and Arts, Sakarya University, Sakarya, Turkey, ORCID: 0000-0003-2242-8940

${ }^{2}$ Department of Mathematics, Faculty of Science and Arts, Sakarya University, Sakarya, Turkey, ORCID: 0000-0002-7183-7081

*Corresponding author: sersoy@sakarya.edu.tr

Received: 22 August 2021, Accepted: 1 October 2021, Available online: 1 October 2021

\section{Introduction}

J. Cockle introduced commutative quaternions as Tessarine numbers in $[10,11,12]$. Besides C. Segre studied these numbers by denominating them bicomplex numbers [3]. Afterwards, G. B. Price comprehensively analyzed bicomplex numbers, functions defined by bicomplex power series, derivatives, integrals, holomorphic functions and also their generalizations to higher dimensions [7]. Actually, the system of bicomplex numbers (Tessarine numbers) is a special case of the commutative fourcomplex numbers system that was generalized by F. Catoni et al. in [6]. The set of generalized commutative quaternions is defined as

$$
\{q \mid q=t+\mathrm{i} x+\mathrm{j} y+\mathrm{k} z ; t, x, y, z \in \mathbb{R}\}
$$

where $\mathrm{i}^{2}=\mathrm{k}^{2}=\alpha, \mathrm{j}^{2}=1, \mathrm{ij}=\mathrm{ji}=\mathrm{k}$. A generalized commutative quaternion is called an elliptic, parabolic or hyperbolic commutative quaternion, respectively; provided that $\alpha<0, \alpha=0$ or $\alpha>0$. In the case of $\alpha=-1$, the elliptic quaternions corresponds to bicomplex numbers. However, the case of $\alpha=1$ has not been handled as well as the bicomplex case. In the meantime, the commutative quaternions and their higher versions were considered by S. Olariu and in the case of $\alpha=1$, a commutative quaternion was called hyperbolic fourcomplex number in [20]. Recently, the set of zeros of polynomials of hyperbolic fourcomplex numbers were studied and these numbers were denominated bihyperbolic numbers since they can be written as a pair of hyperbolic numbers [1].

On the other hand, the hyperbolic fourcomplex numbers are used in digital signal processing and these numbers are called 
multi-hyperbolic numbers [4]. Also, multi-hyperbolic numbers are a generalization of the hyperbolic fourcomplex numbers, since multi-hyperbolic numbers include the hyperbolic fourcomplex numbers.

Apart from all these, detailed surveys on the algebraic [13], geometric and topological [14], and combinatorial properties $[8,9]$ of bihyperbolic numbers were given. However, bihyperbolic modules and topological bihyperbolic modules have not investigated yet.

The real or complex vector space, topological vector space and balanced, convex and absorbing sets in these spaces are known very well in the literature $[2,21]$. These concepts are thought again with the discovery of the quaternions and especially commutative quaternions. For instance, the bicomplex modules are introduced with the discovery of bicomplex numbers. The set of bicomplex numbers is a commutative ring. Hence, the researches on modules over this ring are accelerated with new results on commutative algebra $[5,16]$. Also, topological bicomplex modules are presented and balanced, convex and absorbing sets are investigated in these modules [17, 18].

As its known, the set of hyperbolic numbers is a subalgebra of the algebra of bicomplex numbers and the system of hyperbolic numbers is an active studying area in several disciplines. Besides, hyperbolic module and convex set in this module partially are studied in [15]. In connection with these, we introduce hyperbolic modules, bihyperbolic modules, topological hyperbolic modules and topological bihyperbolic modules. Also, we give new results on these subjects by using the idempotent representations of bihyperbolic numbers which were analyzed in detail [13, 14].

\section{Preliminaries}

Definition 2.1. The set of bihyperbolic numbers is defined as

$$
H_{2}=\left\{\zeta \mid \zeta=z_{1}+j_{2} z_{2}, \quad z_{1}, z_{2} \in H\left(j_{1}\right)\right\}
$$

where $j_{1}, j_{2}$ are hyperbolic units satisfying $j_{1} j_{2}=j_{2} j_{1}=j_{3}, j_{s}^{2}=1, j_{s} \neq \pm 1$ for $s=1,2,3$ and $H\left(j_{1}\right)=\left\{z \mid z=x+j_{1} y: x, y \in \mathbb{R}\right\}$ is the set of hyperbolic numbers based on hyperbolic unit $j_{1}$ [13].

Definition 2.2. The set of multi-hyperbolic numbers is given by

$$
H_{n}=\left\{A+j_{n} B \mid A, B \in H_{n-1}, j_{n}^{2}=1, j_{n} \neq \pm 1\right\}
$$

for $n \in \mathbb{Z}^{+}$.

The set $H_{0}$ is the real numbers set and the set $H_{1}$ is the hyperbolic numbers set corresponding $H\left(\mathrm{j}_{1}\right)$ in the previous definition. In the rest of the article, the notion $H$ will be used for the hyperbolic numbers set based on the hyperbolic unit $\mathrm{j}_{1}$.

The space, null, and time cones of $z_{0} \in H$ are defined as

$$
\begin{gathered}
S H\left(z_{0}\right)=\left\{z \in H \mid\left(z-z_{0}\right) \overline{\left(z-z_{0}\right)}>0 \text { or } z=z_{0}\right\}, \\
N H\left(z_{0}\right)=\left\{z \in H \mid\left(z-z_{0}\right) \overline{\left(z-z_{0}\right)}=0\right\},
\end{gathered}
$$

and

$$
T H\left(z_{0}\right)=\left\{z \in H \mid\left(z-z_{0}\right) \overline{\left(z-z_{0}\right)}<0 \text { or } z=z_{0}\right\}
$$

respectively [14].

Although the sets $\mathrm{H}$ and $\mathrm{H}_{2}$ are commutative rings with unity according to the addition and multiplication operations, they do not have field structure algebraically since they have non-invertible elements according to multiplication operation.

There are especially non-invertible elements such as

$$
e_{1, \mathrm{j}_{s}}=\frac{1+\mathrm{j}_{s}}{2} \text { and } e_{2, \mathrm{j}_{s}}=\frac{1-\mathrm{j}_{s}}{2} \text { for } s=1,2,3
$$

These numbers are hyperbolic numbers with the hyperbolic units $\mathrm{j}_{s}$ and they are called idempotent elements because of $\left(e_{1, \mathrm{j}_{s}}\right)^{n}=e_{1, \mathrm{j}_{s}}$ and $\left(e_{2, \mathrm{j}_{s}}\right)^{n}=e_{2, \mathrm{j}_{s}}$ for $n \in \mathbb{Z}^{+}[13]$. Every element of $H_{2}$ can be written as a linear decomposition of the set 
$\left\{e_{1, \mathrm{j}_{s}}, e_{2, \mathrm{j}_{s}}\right\}$ in three different ways which are $\zeta=\zeta_{1, \mathrm{j}_{s}} e_{1, \mathrm{j}_{s}}+\zeta_{2, \mathrm{j}_{s}} e_{2, \mathrm{j}_{s}}$ for $\zeta \in H_{2}$ with $s=1,2,3$. The coefficients of the linear decompositions of a bihyperbolic number are bihyperbolic numbers for $s=1$ and hyperbolic numbers based on the hyperbolic unit $\mathrm{j}_{1}$ for $s=2,3$. These representations are given for $s=1,2$ in [13] and for $s=3$ in [6]. More details about the idempotent representations of bihyperbolic numbers can be found in $[13,14]$.

There is another idempotent representation of bihyperbolic numbers in the literature. Briefly, a bihyperbolic number $\zeta=x_{0}+\mathrm{j}_{1} x_{1}+\mathrm{j}_{2} x_{2}+\mathrm{j}_{3} x_{3}$ can be written as $\zeta=w_{1} i_{1}+w_{2} i_{2}+w_{3} i_{3}+w_{4} i_{4}$ where $i_{1}, i_{2}, i_{3}$ and $i_{4}$ are bihyperbolic components such that $i_{1}=\frac{1+\mathrm{j}_{1}+\mathrm{j}_{2}+\mathrm{j}_{3}}{4}, i_{2}=\frac{1-\mathrm{j}_{1}+\mathrm{j}_{2}-\mathrm{j}_{3}}{4}, i_{3}=\frac{1+\mathrm{j}_{1}-\mathrm{j}_{2}-\mathrm{j}_{3}}{4}, i_{4}=\frac{1-\mathrm{j}_{1}-\mathrm{j}_{2}+\mathrm{j}_{3}}{4}$ and $w_{1}=x_{0}+x_{1}+x_{2}+x_{3}, w_{2}=x_{0}-x_{1}+x_{2}-x_{3}$, $w_{3}=x_{0}+x_{1}-x_{2}-x_{3}$ and $w_{4}=x_{0}-x_{1}-x_{2}+x_{3}$ where $x_{0}, x_{1}, x_{2}, x_{3} \in \mathbb{R}$ [20]. Hence, a partial order is defined on the real vector space $H_{2}$ by using this representation in [13]. It defines as $\zeta \leq \varphi$ for $\zeta, \varphi \in H_{2}$ if and only if $w_{k} \leq \tilde{w}_{k}$ where $\zeta=w_{k} i_{k}$ and $\varphi=\tilde{w}_{k} i_{k}$ for $k=1,2,3,4$ [13]. Moreover, positive bihyperbolic numbers set is given with this partial order such that $H_{2}^{+}=\left\{\zeta \mid \zeta=w_{k} i_{k}, w_{k} \geq 0\right\}$ [13]. Also, positive hyperbolic numbers are known in the literature such that $H^{+}=\left\{z \mid z=x+\mathrm{j}_{1} y=(x+y) e_{1, \mathrm{j}_{1}}+(x-y) e_{2, \mathrm{j}_{1}}, x+y \geq 0, x-y \geq 0\right\}[5]$.

On the other hand, a bihyperbolic number $\zeta=x_{0}+\mathrm{j}_{1} x_{1}+\mathrm{j}_{2} x_{2}+\mathrm{j}_{3} x_{3}$ has three conjugates such that $\bar{\zeta}^{\mathrm{j}_{1}}=x_{0}+\mathrm{j}_{1} x_{1}-\mathrm{j}_{2} x_{2}-\mathrm{j}_{3} x_{3}, \bar{\zeta}^{\mathrm{j}_{2}}=x_{0}-\mathrm{j}_{1} x_{1}+\mathrm{j}_{2} x_{2}-\mathrm{j}_{3} x_{3}$ and $\bar{\zeta}^{\mathrm{j}_{3}}=x_{0}-\mathrm{j}_{1} x_{1}-\mathrm{j}_{2} x_{2}+\mathrm{j}_{3} x_{3}$ [6]. Considering these conjugates, the hyperbolic valued modulus is introduced [9]. It is defined as $|\zeta|_{\mathrm{j}_{s}}=\sqrt{\mid \zeta \bar{\zeta}^{\mathrm{j}_{s}}} \mid$ for $s=1,2,3$ and named $\mathrm{j}_{s}$-modulus of $\zeta$. Also, by taking $x_{0} x_{1}-x_{2} x_{3}=0, x_{0} x_{2}-x_{1} x_{3}=0$ and $x_{0} x_{3}-x_{1} x_{2}=0$, three different hypersurfaces of $H_{2}$ are defined such that

$$
\begin{aligned}
& M_{1}=\left\{x_{0}+\mathrm{j}_{1} x_{1}+\mathrm{j}_{2} x_{2}+\mathrm{j}_{3} x_{3} \mid x_{0} x_{1}-x_{2} x_{3}=0\right\} \\
& M_{2}=\left\{x_{0}+\mathrm{j}_{1} x_{1}+\mathrm{j}_{2} x_{2}+\mathrm{j}_{3} x_{3} \mid x_{0} x_{2}-x_{1} x_{3}=0\right\}
\end{aligned}
$$

and

$$
M_{3}=\left\{x_{0}+\mathrm{j}_{1} x_{1}+\mathrm{j}_{2} x_{2}+\mathrm{j}_{3} x_{3} \mid x_{0} x_{3}-x_{1} x_{2}=0\right\}
$$

The modulus of $\zeta$ is given by

$$
\begin{aligned}
& |\zeta|_{\mathrm{j}_{1}}=\sqrt{\left|x_{0}{ }^{2}+x_{1}{ }^{2}-x_{2}{ }^{2}-x_{3}{ }^{2}\right|} \\
& |\zeta|_{\mathrm{j}_{2}}=\sqrt{\left|x_{0}^{2}-x_{1}{ }^{2}+x_{2}{ }^{2}-x_{3}{ }^{2}\right|}
\end{aligned}
$$

and

$$
|\zeta|_{j_{3}}=\sqrt{\left|x_{0}^{2}-x_{1}^{2}-x_{2}^{2}+x_{3}^{2}\right|}
$$

in $M_{1}, M_{2}$ and $M_{3}$, respectively [13]. The cones of a bihyperbolic number $\zeta_{0} \in M_{k} \subseteq H_{2}$ are classified as

$$
\begin{gathered}
S M_{k}\left(\zeta_{0}\right)=\left\{\zeta \in M_{k} \mid\left(\zeta-\zeta_{0}\right){\overline{\left(\zeta-\zeta_{0}\right.}}^{\mathrm{j}_{k}}>0 \text { or } \zeta=\zeta_{0}\right\} \\
N M_{k}\left(\zeta_{0}\right)=\left\{\zeta \in M_{k} \mid\left(\zeta-\zeta_{0}\right) \overline{\left(\zeta-\zeta_{0}\right.}{ }^{\mathrm{j}_{k}}=0\right\} \\
\left.T M_{k}\left(\zeta_{0}\right)=\left\{\zeta \in M_{k} \mid\left(\zeta-\zeta_{0}\right) \overline{\left(\zeta-\zeta_{0}\right.}\right)^{\mathrm{j}_{k}}<0 \text { or } \zeta=\zeta_{0}\right\}
\end{gathered}
$$

and they are called space cone, null cone, and time cone for $k=1,2,3$, respectively [14].

Definition 2.3. Let $X$ be a vector space over a field $F$ (real or complex numbers set) and $\varnothing \neq A \subseteq X$ be a subset. If $\lambda x \in A$ or $\lambda A \subseteq A$ where $\lambda A:=\{\lambda x \mid x \in A\}$ for every $x \in A$ and every $\lambda \in F$ with $|\lambda| \leq 1$, then $A$ is balanced (circled) set [19].

Definition 2.4. Let $X$ be a vector space over the real numbers field $\mathbb{R}$ and $\varnothing \neq A \subseteq X$. A is convex if the line segment connecting $x$ and $y$ is included in $A$ for all $x, y \in A$. This means that $(1-t) x+$ ty $\in A$ for $0 \leq t \leq 1$ [19].

Definition 2.5. Let $X$ be a vector space over a field $F$ (real or complex numbers set) and $\varnothing \neq A \subseteq X$. A is absorbing set, if some real number $\lambda>0$ for all $x \in X, x \in \mu A$ for all scalars $\mu \in F$ that is $|\mu| \geq \lambda$ where $\mu A:=\{\mu a \mid a \in A\}$ [19]. 


\section{Topological Hyperbolic Modules}

Definition 3.1. Let $(X, \oplus)$ be a commutative group. If the operations

$$
\begin{aligned}
\oplus: X \times X & \rightarrow X \quad \text { and } & \odot: H \times X & \rightarrow X \\
(u, v) & \rightarrow u+v & (z, u) & \rightarrow z \odot u
\end{aligned}
$$

satisfy the properties

$$
\begin{aligned}
\left(z_{1} z_{2}\right) \odot u & =z_{1} \odot\left(z_{2} \odot u\right), \\
\left(z_{1}+z_{2}\right) \odot u & =\left(z_{1} \odot u\right) \oplus\left(z_{2} \odot u\right), \\
z_{1} \odot(u \oplus v) & =\left(z_{1} \odot u\right) \oplus\left(z_{1} \odot v\right), \\
1_{H} \odot u & =u,\left(1_{H}=1+j_{1} 0=1\right)
\end{aligned}
$$

for every $z_{1}, z_{2} \in H$ and every $u, v \in X$, then $(X, H, \oplus, \odot,+, \cdot)$ is called $H$-module. Later on, $z \odot u$ will be denoted by $z u$.

Example 3.2. Hyperbolic numbers set $H$, bihyperbolic numbers set $H_{2}$ and multi-hyperbolic numbers set $H_{n}$ for $n \in \mathbb{Z}^{+}$are H-modules.

Remark 3.3. Real numbers set $\mathbb{R}$ is not $H$-module because of $H \times \mathbb{R} \rightarrow H$.

Since hyperbolic numbers set $H$ includes the isotropic numbers, the unit balls in $H$ can be classified into three types. So, let us define a new three types of balanced sets by considering three different cases for each hyperbolic number $\lambda=\lambda_{1}+\mathrm{j}_{1} \lambda_{2} \in H$ satisfying $|\lambda|_{H}=\sqrt{|\lambda \bar{\lambda}|}=\sqrt{\left|\lambda_{1}^{2}-\lambda_{2}^{2}\right|} \leq 1$.

Definition 3.4. Let $X$ be $a H-$ module, $\varnothing \neq B \subseteq X$ and $\lambda=\lambda_{1}+j_{1} \lambda_{2} \in H$.

i) $B$ is called $S H$-balanced set if $\lambda B \subseteq B$ for every $\lambda \in S H(O)$ such that $\lambda_{1}^{2}-\lambda_{2}^{2} \leq 1$,

ii) $B$ is called $N H$-balanced set if $\lambda B \subseteq B$ for every $\lambda \in N H(O)$ that is $\lambda_{1}^{2}-\lambda_{2}^{2}=0$,

iii) $B$ is called $T H$-balanced set if $\lambda B \subseteq B$ for every $\lambda \in T H(O)$ such that $-1 \leq \lambda_{1}^{2}-\lambda_{2}^{2}$.

Here, $S H(O), N H(O)$ and $T H(O)$ denotes the space cone, the light cone and the time cone of $H$ at the origin, respectively.

Example 3.5. The subsets $S H(O)$ and $T H(O)$ in $H$-module $H$ are $S H$-balanced sets. But, they are not $N H-b a l a n c e d ~ s e t$ and $T H$-balanced set. Also, the subset $N H(O) \subseteq H$ is $T H, N H$ and $S H$-balanced set.

The partial order on the real vector space $H_{2}$ was introduced in [13]. The definition of $H$-convex set is given in [15] by using such an order as follows: Let $X$ be a $H$-module and $\varnothing \neq B \subseteq X$. If $\lambda x+(1-\lambda) y \in B$ for every $x, y \in B$ and $\lambda \in H^{+}$ with $0 \leq \lambda \leq 1$, then $B$ is called $H$-convex set. Nevertheless, here we investigate especially the $H$-module $H$. Eventually, three different definitions of convex sets which are geometrically meaningful will be given in $H$-module $H$ for the first time as follows.

Definition 3.6. Let $B$ be a non-empty subset of $H$-module $H$. For all $x, y \in B$ and all $\lambda \in \mathbb{R}$ with $0 \leq \lambda \leq 1$,

i) $B$ is called $S H-$ convex set if $y \in S H(x)$ and $\lambda x+(1-\lambda) y \in B$,

ii) $B$ is called $N H-$ convex set if $y \in N H(x)$ and $\lambda x+(1-\lambda) y \in B$,

iii) $B$ is called $T H-$ convex set if $y \in T H(x)$ and $\lambda x+(1-\lambda) y \in B$.

This definition indicates that the classical definition of the convexity is valid for the convexity of a subset of the hyperbolic numbers set. However, three different convexity types are needed depending on whether the line segments connecting all two different elements of the set belong to either the space cone, the light cone or the time cone.

Definition 3.7. Let $X$ be a $H$-module and $\varnothing \neq B \subseteq X$. For all $x \in X$,

i) $B$ is called $S H$-absorbing set if there is a non-negative real number $\lambda$ such that $x \in \mu B$ for all $\mu \in S H(O) \subseteq H$ with $|\mu|_{H} \geq \lambda$ 
ii) $B$ is called TH-absorbing set if there is a non-negative real number $\lambda$ such that $x \in \mu B$ for all $\mu \in T H(O) \subseteq H$ with $|\mu|_{H} \geq \lambda$.

Definition 3.8. Let $X$ be a H-module and $\tau$ is a Hausdorff topology on X. If the operations

$$
\begin{aligned}
& +: X \times X \rightarrow X \\
& \cdot: H \times X \rightarrow X
\end{aligned}
$$

are continuous, then the pair $(X, \tau)$ is called a topological hyperbolic module or topological $H$-module.

\section{Topological Bihyperbolic Modules}

Since $\left(H_{2},+, \cdot\right)$ is a commutative ring with unity, we can construct a module structure over this ring. For instance, the bihyperbolic numbers set $H_{2}$ or the multi-hyperbolic numbers set $H_{n}$ for $n \in\{2,3,4, \ldots\}$ are $H_{2}$-modules.

Let $X$ be an arbitrary $\mathrm{H}_{2}$-module with the classical addition and multiplication operations. The idempotent representations of the elements of $X$ are given correlatively the elements of $H_{2}$ in the following theorem.

Theorem 4.1. Let $X$ be a $H_{2}$-module. Then $X=e_{1, j_{s}} X+e_{2, j_{s}} X$ for $s=1,2,3$.

Proof. Let $x \in X$. Then $e_{1, \mathrm{j}_{s}}+e_{2, \mathrm{j}_{s}}=1$ for $e_{1, \mathrm{j}_{s}}, e_{2, \mathrm{j}_{s}} \in H\left(\mathrm{j}_{s}\right) \subseteq H_{2}$ and $s=1,2,3$. Hence, the element $x$ can be written as

$$
x=\left(e_{1, \mathrm{j}_{s}}+e_{2, \mathrm{j}_{s}}\right) x=e_{1, \mathrm{j}_{s}} x+e_{2, \mathrm{j}_{s}} x .
$$

Since each element of $X$ can be written as above, it can be generalized to the whole set.

Here if we write $e_{1, \mathrm{j}_{s}} X=X_{1, \mathrm{j}_{s}}$ and $e_{2, \mathrm{j}_{s}} X=X_{2, \mathrm{j}_{s}}$, then $X=X_{1, \mathrm{j}_{s}}+X_{2, \mathrm{j}_{s}}$.

Corollary 4.2. Let $X$ be a $H_{2}$-module. Then, there are $e_{1, j_{s}} X=e_{1, j_{s}} X_{1, j_{s}}$ and $e_{2, j_{s}} X=e_{2, j_{s}} X_{2, j_{s}}$ equations for $s=1,2,3$.

Proof. Let $e_{1, \mathrm{j}_{s}} X=X_{1, \mathrm{j}_{s}}$. Then multiplying both sides of this equation from left by $e_{1, \mathrm{j}_{s}}$ gives us $e_{1, \mathrm{j}_{s}}\left(e_{1, \mathrm{j}_{s}} X\right)=e_{1, \mathrm{j}_{s}} X_{1, \mathrm{j}_{s}}$. Hence $e_{1, \mathrm{j}_{s}} X=e_{1, \mathrm{j}_{s}} X_{1, \mathrm{j}_{s}}$, since $e_{1, \mathrm{j}_{s}}$ and $e_{2, \mathrm{j}_{s}}$ are the idempotent elements. Similarly, we can write $e_{2, \mathrm{j}_{s}}\left(e_{2, \mathrm{j}_{s}} X\right)=e_{2, \mathrm{j}_{s}} X_{2, \mathrm{j}_{s}}$ whenever $e_{2, j_{s}} X=X_{2, \mathrm{j}_{s}}$. So, $e_{2, \mathrm{j}_{s}} X=e_{2, \mathrm{j}_{s}} X_{2, \mathrm{j}_{s}}$ is obtained.

Corollary 4.3. Let $X$ be a $H_{2}$-module. Then, $X=e_{1, j_{s}} X_{1, j_{s}}+e_{2, j_{s}} X_{2, j_{s}}$ for $s=1,2,3$.

Corollary 4.4. Let $X$ be a $H_{2}$-module. Then, $X_{1, j_{s}}$ and $X_{2, j_{s}}$ are $H_{2}$-submodules of $X$ for $s=1,2,3$.

Proof. Let $X$ be a $H_{2}$-module and $X_{1, \mathrm{j}_{s}} \subseteq X$ for $s=1,2,3$. Moreover, let $t_{1}, t_{2} \in X_{1, \mathrm{j}_{s}}$. There are the elements $x$ and $y$ in $X$ satisfied the equations $t_{1}=e_{1, \mathrm{j}_{s}} x$ and $t_{2}=e_{1, \mathrm{j}_{s}} y$, since $X_{1, \mathrm{j}_{s}}=e_{1, \mathrm{j}_{s}} X .(X,+)$ is a commutative group, since $X$ is a $H_{2}$-module. Hence, $x-y \in X$. So, $t_{1}-t_{2}=e_{1, j_{s}} x-e_{1, j_{s}} y=e_{1, j_{s}}(x-y) \in e_{1, j_{s}} X=X_{1, j_{s}}$. On the other hand, let $\zeta \in H_{2}$ and $t \in X_{1, \mathrm{j}_{s}}$. The product of $\zeta$ and $t$ is $\zeta t=\left(\zeta_{1, \mathrm{j}_{s}} e_{1, \mathrm{j}_{s}}+\zeta_{2, \mathrm{j}_{s}} e_{2, \mathrm{j}_{s}}\right)\left(e_{1, \mathrm{j}_{s}} x\right)=\zeta_{1, \mathrm{j}_{s}} e_{1, \mathrm{j}_{s}} x$ and $\zeta_{1, \mathrm{j}_{s}} x \in X$ since $X$ a $H_{2}-$ module. Hence $\zeta t=e_{1, \mathrm{j}_{s}} \zeta_{1, \mathrm{j}_{s}} x \in e_{1, \mathrm{j}_{s}} X=X_{1, \mathrm{j}_{s}}$. Consequently, $X_{1, \mathrm{j}_{s}}$ is a $H_{2}$-submodule of the $H_{2}$-module $X$. Similarly, the set $X_{2, \mathrm{j}_{s}}$ is a $H_{2}$-submodule of the $H_{2}$-module $X$.

Especially, the subsets $X_{1, \mathrm{j}_{s}}$ and $X_{2, \mathrm{j}_{s}}$ are $H$-submodules of the $H_{2}$-module $X$ for $s=2,3$ since $\zeta_{1, \mathrm{j}_{s}}, \zeta_{2, \mathrm{j}_{s}} \in H$.

Corollary 4.5. The subsets $e_{1, j_{s}} H_{2}$ and $e_{2, j_{s}} H_{2}$ are $H_{2}$-modules for $s=1,2,3$. Especially, these sets are $H$-modules for $s=2,3$.

Definition 4.6. Let $X$ be a $H_{2}$-module. If there is a finite $H_{2}$-base such that $\left\{x_{l}: l=1, \ldots, n\right\} \subseteq X$, then $X$ is a free $H_{2}$-module. The free $H_{2}-$ module $X$ can be written as $X=\left\{x \mid x=\sum_{l=1}^{n} \zeta_{l} x_{l}, \zeta_{l} \in H_{2}, x_{l} \in X\right\}$.

Definition 4.7. Let $\mathrm{X}$ be a free $\mathrm{H}_{2}-$ module.

$$
A:=\left\{\tilde{x} \mid \tilde{x}=\sum_{l=1}^{n} \zeta_{l} x_{l}, \zeta_{l} \in H, x_{l} \in X\right\} \subseteq X
$$

is a free $H$-module depending on the $H_{2}-$ base of $X$. 
Here, when the elements of any subset $A$ of the free $H_{2}$-module $X$ are written as a linear combination of the finite base $\left\{x_{l}: l=1, \ldots, n\right\} \subseteq X$, if the coefficients are bihyperbolic number, then the subset $A$ is a free $H_{2}$-module depends on the $\mathrm{H}_{2}$-base of $X$.

Example 4.8. Each element of $\mathrm{H}_{2}$ can be written as a linear combination of the idempotent elements $e_{1, j_{s}}$ and $e_{2, j_{s}}$ for $s=1,2,3$ such that $\zeta=\zeta_{1, j_{s}} e_{1, j_{s}}+\zeta_{2, j_{s}} e_{2, j_{s}} \in H_{2}$. Also, the set $\left\{e_{1, j_{s}}, e_{2, j_{s}}\right\}$ is linearly independent. Therefore, the subset $\left\{e_{1, j_{s}}, e_{2, j_{s}}\right\} \subseteq H_{2}$ is a base of the $H_{2}$. It is known that $\zeta_{1, j_{s}}, \zeta_{2, j_{s}} \in H_{2}$ for $s=1$ and $\zeta_{1, j_{s}}, \zeta_{2, j_{s}} \in H$ for $s=2,3$. So, $H_{2}$ is a free $H_{2}-$ module for $s=1$. Moreover, $H_{2}$ is a free $H$-module according to $H_{2}$-base for $s=2,3$.

Now, let us give the necessary conditions for any subset of a $\mathrm{H}_{2}$-module to be balanced, convex or absorbing set. In order to give the conditions specified here, there must be a real-valued norm on the ring in which the module structure is defined. Since there are real-valued norms on the hypersurfaces $M_{k} \subseteq H_{2}$ for $k=1,2,3$, related conditions will be given and theorems will be proved by using the elements of $M_{k}$.

Three different balanced (circular) sets, convex sets and two different absorbing (swallowing) sets have emerged on the $H_{2}$-module due to the presence of light cone on hypersurfaces $M_{k} \subseteq H_{2}$.

Firstly, the following definition of a balanced (circular) set is given by considering the three different conditions for each bihyperbolic number $\zeta \in M_{k} \subseteq H_{2}$ satisfying the condition $|\zeta|_{\mathrm{j}_{k}}=\sqrt{\left|\zeta \bar{\zeta}^{\mathrm{j}_{k}}\right|} \leq 1$.

Definition 4.9. Let $X$ be a $H_{2}-$ module, $\varnothing \neq B \subseteq X$ and $\zeta \in M_{k} \subseteq H_{2}(k=1,2,3)$.

i) $B$ is called $S M_{k}$-balanced set if $\zeta B \subseteq B$ for every $\zeta \in S M_{k}(O)$ such that $\zeta \bar{\zeta}^{j_{k}} \leq 1$,

ii) $B$ is called $N M_{k}$-balanced set if $\zeta B \subseteq B$ for every $\zeta \in N M_{k}(O)$ such that $\zeta \bar{\zeta}^{j_{k}}=0$,

iii) $B$ is called $T M_{k}$-balanced set if $\zeta B \subseteq B$ for every $\zeta \in T M_{k}(O)$ such that $-1 \leq \zeta \bar{\zeta}^{j_{k}}$.

Here the sets $S_{k}(O), N M_{k}(O)$ and $T M_{k}(O)$ are the space cone, the null cone and the time cone at the origin in the hypersurfaces $M_{k}$, respectively.

Theorem 4.10. Let $X$ be a $H_{2}$-module and the set $B$ is a $S M_{k}$-balanced or $T M_{k}$-balanced subset of $X$ for $k=1,2,3$.

i) $\zeta B=B$ for every $\zeta \in M_{k} \subseteq H_{2}$ such that $|\zeta|_{j_{k}}=1$.

ii) $\zeta B=|\zeta|_{j_{k}}$ B for every $\zeta \in M_{k} \subseteq H_{2}$ such that $|\zeta|_{j_{k}} \neq 0$.

Proof. i) Let $\zeta \in M_{k}$ such that $|\zeta|_{\mathrm{j}_{k}}=1$. Since $B$ is a $S M_{k}$-balanced or $T M_{k}$-balanced set, $\zeta B \subseteq B$. On the other hand

$$
\left|\frac{1}{\zeta}\right|_{\mathrm{j}_{k}}=\frac{1}{|\zeta|_{\mathrm{j}_{k}}}=1
$$

So $\frac{1}{\zeta} B \subseteq B$ and in this way $B \subseteq \zeta B$. Consequently $\zeta B=B$.

ii) Let's take any $\zeta \in M_{k}$ such that $|\zeta|_{\mathrm{j}_{k}} \neq 0$. Then

$$
\left|\frac{\zeta}{|\zeta|_{\mathrm{j}_{k}}}\right|_{\mathrm{j}_{k}}=1
$$

So,

$$
\frac{\zeta}{|\zeta|_{\mathrm{j}_{k}}} B=B
$$

from the condition (i). Hence, we have $\zeta B=|\zeta|_{\mathrm{j}_{k}} B$.

Theorem 4.11. Let $X$ be a $H_{2}$-module and the set $B$ is a $S M_{k}$-balanced subset of $X$ for $k=1,2,3$. 
i) For $s=k=1, e_{1, j_{s}} B=B_{1, j_{s}}$ and $e_{2, j_{s}} B=B_{2, j_{s}}$ are $S M_{k}$-balanced subsets of $H_{2}$-modules $e_{1, j_{s}} X=X_{1, j_{s}}$ and $e_{2, j_{s}} X=X_{2, j_{s}}$, respectively.

ii) For $s, k=2,3$ and $s=k, e_{1, j_{s}} B=B_{1, j_{s}}$ and $e_{2, j_{s}} B=B_{2, j_{s}}$ are $S H$-balanced subsets of $H$-modules $e_{1, j_{s}} X=X_{1, j_{s}}$ and $e_{2, j_{s}} X=X_{2, j_{s}}$, respectively.

Proof. $\quad$ i) Let $X$ be a $H_{2}$-module and $B$ be a $S M_{k}$-balanced subset of $X$ for $k=1$. Therefore, $\zeta x \in B$ for all $x \in B$ and all $\zeta \in S M_{k}(O)$ such that $\zeta \bar{\zeta}^{j_{k}} \leq 1$. Assume that the idempotent representation of $\zeta$ is $\zeta=\zeta_{1, \mathrm{j}_{s}} e_{1, \mathrm{j}_{s}}+\zeta_{2, \mathrm{j}_{s}} e_{2, \mathrm{j}_{s}}$ for $s=1$.

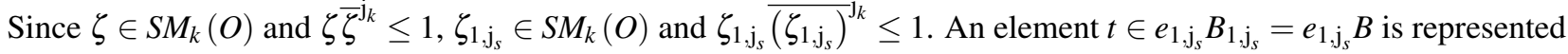
by $t=e_{1, j_{s}} x$ for $x \in B$.

Hence, $\zeta_{1, \mathrm{j}_{s}} t=\zeta_{1, \mathrm{j}_{s}} e_{1, \mathrm{j}_{s}} x=e_{1, \mathrm{j}_{s}} \zeta_{1, \mathrm{j}_{s}} x=e_{1, \mathrm{j}_{s}} \zeta x \in e_{1, \mathrm{j}_{s}} B=e_{1, \mathrm{j}_{s}} B_{1, \mathrm{j}_{s}}$ where $e_{1, \mathrm{j}_{s}} \zeta=e_{1, \mathrm{j}_{s}}\left(\zeta_{1, \mathrm{j}_{s}} e_{1, \mathrm{j}_{s}}+\zeta_{2, \mathrm{j}_{s}} e_{2, \mathrm{j}_{s}}\right)=e_{1, \mathrm{j}_{s}} \zeta_{1, \mathrm{j}_{s}}$. So, the set $e_{1, \mathrm{j}_{s}} B_{1, \mathrm{j}_{s}}$ is $S M_{k}$-balanced set of the $H_{2}$-module $e_{1, \mathrm{j}_{s}} X_{1, \mathrm{j}_{s}}$. Similarly, the set $e_{2, \mathrm{j}_{s}} B=B_{2, \mathrm{j}_{s}}$ is a $S M_{k}$-balanced set of the $H_{2}$-module $e_{2, \mathrm{j}_{s}} X=X_{2, \mathrm{j}_{s}}$ for $s=k=1$.

ii) Let $X$ be a $H_{2}$-module and $B$ be a $S M_{k}$-balanced subset of $X$ for $k=2,3$. Hence, $\zeta x \in B$ for all $x \in B$ and all $\zeta \in S M_{k}(O)$ such that $\zeta \bar{\zeta}^{\mathrm{j}_{k}} \leq 1$. The idempotent representation of $\zeta$ is $\zeta=\zeta_{1, \mathrm{j}_{s}} e_{1, \mathrm{j}_{s}}+\zeta_{2, \mathrm{j}_{s}} e_{2, \mathrm{j}_{s}}$ for $s=2,3$ and $e_{1, \mathrm{j}_{s}} \zeta=e_{1, \mathrm{j}_{s}}\left(\zeta_{1, \mathrm{j}_{s}} e_{1, \mathrm{j}_{s}}+\zeta_{2, \mathrm{j}_{s}} e_{2, \mathrm{j}_{s}}\right)=e_{1, \mathrm{j}_{s}} \zeta_{1, \mathrm{j}_{s}}$. Moreover, the coefficient $\zeta_{1, \mathrm{j}_{s}} \in H \subseteq H_{2}$ is $\zeta_{1, \mathrm{j}_{s}} \in S H(O)$ and it provides the inequality $\zeta_{1, \mathrm{j}_{s}} \zeta_{1, \mathrm{j}_{s}} \leq 1$ for $s, k=2,3 s=k$. An element $t \in e_{1, \mathrm{j}_{s}} B_{1, \mathrm{j}_{s}}=e_{1, \mathrm{j}_{s}} B$ can be written as $t=e_{1, \mathrm{j}_{s}} x$ since $x \in B$. Thus, $\zeta_{1, \mathrm{j}_{s}} t=\zeta_{1, \mathrm{j}_{s}} e_{1, \mathrm{j}_{s}} x=e_{1, \mathrm{j}_{s}} \zeta_{1, \mathrm{j}_{s}} x=e_{1, \mathrm{j}_{s}} \zeta x \in e_{1, \mathrm{j}_{s}} B=e_{1, \mathrm{j}_{s}} B_{1, \mathrm{j}_{s}}$. So, the sets $e_{1, \mathrm{j}_{s}} B_{1, \mathrm{j}_{s}}$ are $S H$-balanced sets of $H$-modules $e_{1, \mathrm{j}_{s}} X_{1, \mathrm{j}_{s}}$ for $s, k=2,3$ and $s=k$. Similarly, the sets $e_{2, \mathrm{j}_{s}} B=B_{2, \mathrm{j}_{s}}$ are $S H$-balanced sets of $H$-modules $e_{2, \mathrm{j}_{s}} X=X_{2, \mathrm{j}_{s}}$ for $s, k=2,3 s=k$.

Theorem 4.12. Let $X$ be a $H_{2}$-module and $B$ be a $N M_{k}$-balanced subset of $X$ for $k=1,2,3$.

i) For $s=k=1, e_{1, j_{s}} B=B_{1, j_{s}}$ and $e_{2, j_{s}} B=B_{2, j_{s}}$ are $N M_{k}$-balanced subsets of $H_{2}$-modules $e_{1, j_{s}} X=X_{1, j_{s}}$ and $e_{2, j_{s}} X=X_{2, j_{s}}$, respectively.

ii) For $s, k=2,3$ and $s=k, e_{1, j_{s}} B=B_{1, j_{s}}$ and $e_{2, j_{s}} B=B_{2, j_{s}}$ are $N H$-balanced subsets of $H$-modules $e_{1, j_{s}} X=X_{1, j_{s}}$ and $e_{2, j_{s}} X=X_{2, j_{s}}$, respectively.

Theorem 4.13. Let $X$ be a $H_{2}$-module and $B$ be a $T M_{k}$-balanced subset of $X$ for $k=1,2,3$.

i) For $s=k=1, e_{1, j_{s}} B=B_{1, j_{s}}$ and $e_{2, j_{s}} B=B_{2, j_{s}}$ are $T M_{k}$-balanced subsets of $H_{2}$-modules $e_{1, j_{s}} X=X_{1, j_{s}}$ and $e_{2, j_{s}} X=X_{2, j_{s}}$, respectively.

ii) For $s, k=2,3$ and $s=k, e_{1, j_{s}} B=B_{1, j_{s}}$ and $e_{2, j_{s}} B=B_{2, j_{s}}$ are $T H$-balanced subsets of $H$-modules $e_{1, j_{s}} X=X_{1, j_{s}}$ and $e_{2, j_{s}} X=X_{2, j_{s}}$, respectively.

Theorem 4.14. Let $X$ be a $H_{2}$-module and $B$ be a $N M_{k}$-balanced subset of $X$ for $k=1,2,3$. Then $e_{1, j_{s}} B=B_{1, j_{s}} \subseteq B$ and $e_{2, j_{s}} B=B_{2, j_{s}} \subseteq B$ for $s=1,2,3$ and $s \neq k$.

Proof. Let $x \in B$ and an element $t \in e_{1, \mathrm{j}_{s}} B_{1, \mathrm{j}_{s}}=e_{1, \mathrm{j}_{s}} B$ be given by $t=e_{1, \mathrm{j}_{s}} x$. Since the set $B$ is $N M_{k}-$ balanced set, $\zeta x \in B$ for all $\zeta \in N M_{k}(O) . e_{1, \mathrm{j}_{s}} \in N M_{k}(O)$ for $s, k=1,2,3$ and $s \neq k$. Thus, if we choose $\zeta=e_{1, \mathrm{j}_{s}}$, then $e_{1, \mathrm{j}_{s}} B_{1, \mathrm{j}_{s}} \subseteq B$. Similarly, if $\zeta=e_{2, \mathrm{j}_{s}}$ is chosen, $e_{2, \mathrm{j}_{s}} B_{2, \mathrm{j}_{s}} \subseteq B$ for $s, k=1,2,3$ and $s \neq k$.

The inclusions $e_{1, \mathrm{j}_{s}} B=B_{1, \mathrm{j}_{s}} \subseteq B$ and $e_{2, \mathrm{j}_{s}} B=B_{2, \mathrm{j}_{s}} \subseteq B$ do not exist for a $S M_{k}$-balanced or $T M_{k}$-balanced subset $B$ of $H_{2}$-modules $X$. Because the idempotent components $e_{1, \mathrm{j}_{s}}$ and $e_{2, \mathrm{j}_{s}}$ are $e_{1, \mathrm{j}_{s}}, e_{2, \mathrm{j}_{s}} \notin M_{k}$ for $s=k$ and $e_{1, \mathrm{j}_{s}}, e_{2, \mathrm{j}_{s}} \in N M_{k}$ for $s \neq k$.

Definition 4.15. Let $X$ be a $H_{2}-$ module and $\varnothing \neq B \subseteq X$. B is a $H_{2}-$ convex set if $\zeta x+(1-\zeta) y \in B$ for all $x, y \in B$ and all $\zeta \in H_{2}^{+}$such that $0 \leq \zeta \leq 1$.

Theorem 4.16. Let $X$ be a $H_{2}$-module and $\varnothing \neq B \subseteq X$ is a $H_{2}$-convex subset of $X$.

i) The sets $e_{1, j_{s}} B$ and $e_{2, j_{s}} B$ are $H_{2}-$ convex sets of $H_{2}-$ modules $e_{1, j_{s}} X$ and $e_{2, j_{s}} X$ for $s=1$, respectively.

ii) The sets $e_{1, j_{s}} B$ and $e_{2, j_{s}} B$ are $H$-convex sets of the $H$-modules $e_{1, j_{s}} X$ and $e_{2, j_{s}} X$ for $s=2,3$, respectively. 
iii) There are the inclusions $e_{1, j_{s}} B \subseteq B$ and $e_{2, j_{s}} B \subseteq B$ for $s=1,2,3$, if $\theta \in B$ where $\theta$ is the unit element of the $H_{2}-$ module $X$.

Proof. i) Let $B$ be a $H_{2}$-convex subset of the $H_{2}$-module $X$ and $t_{1}, t_{2} \in e_{1, \mathrm{j}_{s}} B$ for $s=1$. There exist $x, y \in B$ such that $t_{1}=e_{1, \mathrm{j}_{s}} x \in e_{1, \mathrm{j}_{s}} B$ and $t_{2}=e_{1, \mathrm{j}_{s}} y \in e_{1, \mathrm{j}_{s}} B$. Consider $\zeta=\zeta_{1, \mathrm{j}_{s}} e_{1, \mathrm{j}_{s}}+\zeta_{2, \mathrm{j}_{s}} e_{2, \mathrm{j}_{s}} \in H_{2}^{+}$for all $\zeta_{1, \mathrm{j}_{s}}, \zeta_{2, \mathrm{j}_{s}} \in H_{2}^{+}$such that $\zeta_{1, \mathrm{j}_{s}}, \zeta_{2, \mathrm{j}_{s}} \in[0,1]$. If $\zeta_{1, \mathrm{j}_{s}}, \zeta_{2, \mathrm{j}_{s}} \in[0,1]$, then $\zeta \in[0,1][13]$. Thus, since the set $B$ is $H_{2}$-convex, $\zeta x+(1-\zeta) y \in B$ for $x, y \in B, \zeta \in H_{2}^{+}$and $\zeta \in[0,1]$. In that case,

$$
\begin{aligned}
e_{1, \mathrm{j}_{s}}(\zeta x+(1-\zeta) y) & =e_{1, \mathrm{j}_{s}}\left(\left(\zeta_{1, \mathrm{j}_{s}} e_{1, \mathrm{j}_{s}}+\zeta_{2, \mathrm{j}_{s}} e_{2, \mathrm{j}_{s}}\right) x\right. \\
& \left.+\left(1-\left(\zeta_{1, \mathrm{j}_{s}} e_{1, \mathrm{j}_{s}}+\zeta_{2, \mathrm{j}_{s}} e_{2, \mathrm{j}_{s}}\right)\right) y\right) \\
& =\zeta_{1, \mathrm{j}_{s}} e_{1, \mathrm{j}_{s}} x+\left(1-\zeta_{1, \mathrm{j}_{s}}\right) e_{1, \mathrm{j}_{s}} y \\
& =\zeta_{1, \mathrm{j}_{s}} t_{1}+\left(1-\zeta_{1, \mathrm{j}_{s}}\right) t_{2} \in e_{1, \mathrm{j}_{s}} B .
\end{aligned}
$$

From here, the set $e_{1, \mathrm{j}_{s}} B$ is a $H_{2}$-convex subset of $H_{2}-$ modules $e_{1, \mathrm{j}_{s}} X$. Similarly, it can be proved that the set $e_{2, \mathrm{j}_{s}} B$ is $H_{2}$-convex subset of $H_{2}$-module $e_{2, j_{s}} X$ for $s=1$.

ii) Let $t_{1}=e_{1, \mathrm{j}_{s}} x \in e_{1, \mathrm{j}_{s}} B$ and $t_{2}=e_{1, \mathrm{j}_{s}} y \in e_{1, \mathrm{j}_{s}} B$ for $x, y \in B$ and $s=2,3$. $\zeta=\zeta_{1, \mathrm{j}_{s}} e_{1, \mathrm{j}_{s}}+\zeta_{2, \mathrm{j}_{s}} e_{2, \mathrm{j}_{s}} \in H_{2}^{+}$such that $\zeta_{1, \mathrm{j}_{s}}, \zeta_{2, \mathrm{j}_{s}} \in H^{+}$and $\zeta_{1, \mathrm{j}_{s}}, \zeta_{2, \mathrm{j}_{s}} \in[0,1]$. Hence, $\zeta \in[0,1]$. Since the set $B$ is $H_{2}-$ convex set $\zeta x+(1-\zeta) y \in B$. Similarly, we get

$$
\begin{aligned}
e_{1, \mathrm{j}_{s}}(\zeta x+(1-\zeta) y) & =e_{1, \mathrm{j}_{s}}\left(\left(\zeta_{1, \mathrm{j}_{s}} e_{1, \mathrm{j}_{s}}+\zeta_{2, \mathrm{j}_{s}} e_{2, \mathrm{j}_{s}}\right) x\right. \\
& \left.+\left(1-\left(\zeta_{1, \mathrm{j}} e_{1, \mathrm{j}_{s}}+\zeta_{2, \mathrm{j}_{s}} e_{2, \mathrm{j}_{s}}\right)\right) y\right) \\
& =\zeta_{1, \mathrm{j}_{s}} e_{1, \mathrm{j}_{s}} x+\left(1-\zeta_{1, \mathrm{j}_{s}}\right) e_{1, \mathrm{j}_{s}} y \\
& =\zeta_{1, \mathrm{j}_{s}} t_{1}+\left(1-\zeta_{1, \mathrm{j}_{s}}\right) t_{2} \in e_{1, \mathrm{j}_{s}} B .
\end{aligned}
$$

Hence, the sets $e_{1, \mathrm{j}_{s}} B$ for $s=2,3$ are $H$-convex subsets of $H$-modules $e_{1, \mathrm{j}_{s}} X$. Also, it can be proved that the sets $e_{2, \mathrm{j}_{s}} B$ are $H$-convex subsets of $H$-modules $e_{2, \mathrm{j}_{s}} X$ for $s=2,3$ in a similar manner.

iii) Let $B$ be a $H_{2}$-convex subset of the $H_{2}$-module $X$ and $\theta \in B . t \in e_{1, \mathrm{j}_{s}} B$ for $s=1,2,3$. There is an element $x \in B$ such that $t=e_{1, \mathrm{j}_{s}} x \in e_{1, \mathrm{j}_{s}} B$. Considering that $\theta \in B$, since $B$ is $H_{2}$-convex subset $e_{1, \mathrm{j}_{s}} x+\left(1-e_{1, \mathrm{j}_{s}}\right) \theta=e_{1, \mathrm{j}_{s}} x=t \in B$ where $0 \leq e_{1, \mathrm{j}_{s}} \leq 1$ and $e_{1, \mathrm{j}_{s}} \in H_{2}^{+}\left(H^{+} \subseteq H_{2}^{+}\right)$for $x, \theta \in B$. Consequently $e_{1, \mathrm{j}_{s}} B \subseteq B$ is obtained. Similarly, we deduce $e_{2, \mathrm{j}_{s}} B \subseteq B$ for $s=1,2,3$.

Lemma 4.17. Let $X$ be a $H_{2}-$ module and the sets $\left\{B_{l}:\right.$ l arbitrary $\}$ be any $H_{2}$-convex subsets of $X$. Then, the set $\cap_{l} B_{l}=B$ is $\mathrm{H}_{2}$-convex, too.

Theorem 4.18. Let $X$ be a $H_{2}$-module and $\varnothing \neq B \subseteq X$ be a $H_{2}$-convex subset. Then, $B=e_{1, j_{s}} B+e_{2, j_{s}} B$ for $s=1,2,3$.

Proof. Assume that $B$ is a $H_{2}$-convex subset of $H_{2}$-modules $X$ and take $x \in B . e_{1, \mathrm{j}_{s}} x \in e_{1, \mathrm{j}_{s}} B$ and $e_{2, \mathrm{j}_{s}} x \in e_{2, \mathrm{j}_{s}} B$ for $s=1,2,3$. Since $e_{1, \mathrm{j}_{s}}+e_{2, \mathrm{j}_{s}}=1$ then

$$
x=\left(e_{1, \mathrm{j}_{s}}+e_{2, \mathrm{j}_{s}}\right) x=e_{1, \mathrm{j}_{s}} x+e_{2, \mathrm{j}_{s}} x \in e_{1, \mathrm{j}_{s}} B+e_{2, \mathrm{j}_{s}} B .
$$

Thus, $B \subseteq e_{1, \mathrm{j}_{s}} B+e_{2, \mathrm{j}_{s}} B$. Conversely, let us take $t_{1} \in e_{1, \mathrm{j}_{s}} B$ and $t_{2} \in e_{2, \mathrm{j}_{s}} B$ where $t_{1}=e_{1, \mathrm{j}_{s}} x$ and $t_{2}=e_{2, \mathrm{j}_{s}} y$ for $x, y \in B$. Since the set $B$ is $H_{2}$-convex, $t_{1}+t_{2}=e_{1, \mathrm{j}_{s}} x+e_{2, \mathrm{j}_{s}} y=e_{1, \mathrm{j}_{s}} x+\left(1-e_{1, \mathrm{j}_{s}}\right) y \in B$ where $e_{1, \mathrm{j}_{s}}, e_{2, \mathrm{j}_{s}} \in H_{2}^{+}$and $0 \leq e_{1, \mathrm{j}_{s}}, e_{2, \mathrm{j}_{s}} \leq 1$. Therefore, $e_{1, \mathrm{j}_{s}} B+e_{2, \mathrm{j}_{s}} B \subseteq B$. This completes the proof.

Theorem 4.19. Let $X$ be $a H_{2}$-module and $\varnothing \neq B \subseteq X$. If the sets $e_{1, j_{s}} B$ and $e_{2, j_{s}} B$ are $H_{2}$-convex sets for $s=1,2,3$ then the set $e_{1, j_{s}} B+e_{2, j_{s}} B$ is a $H_{2}-$ convex subset of $X$, too.

Proof. Assume that $x, y \in e_{1, \mathrm{j}_{s}} B+e_{2, \mathrm{j}_{s}} B$ and $\zeta \in H_{2}^{+}$such that $0 \leq \zeta \leq 1$. Then, $x=e_{1, \mathrm{j}_{s}} x+e_{2, \mathrm{j}_{s}} x$ and $y=e_{1, \mathrm{j}_{s}} y+e_{2, \mathrm{j}_{s}} y$ where $e_{1, \mathrm{j}_{s}} x, e_{1, \mathrm{j}_{s}} y \in e_{1, \mathrm{j}_{s}} B$ and $e_{2, \mathrm{j}_{s}} x, e_{2, \mathrm{j}_{s}} y \in e_{2, \mathrm{j}_{s}} B$. The idempotent representation of $\zeta$ is $\zeta=\zeta_{1, \mathrm{j}_{s}} e_{1, \mathrm{j}_{s}}+\zeta_{2, \mathrm{j}_{s}} e_{2, \mathrm{j}_{s}}$. Hence, $0 \leq \zeta_{1, \mathrm{j}_{s}}, \zeta_{2, \mathrm{j}_{s}} \leq 1$ and $\zeta_{1, \mathrm{j}_{s}}, \zeta_{2, \mathrm{j}_{s}} \in H_{2}^{+}$because of $\zeta \in H_{2}^{+}$. Since the sets $e_{1, \mathrm{j}_{s}} B$ and $e_{2, \mathrm{j}_{s}} B$ are $H_{2}-$ convex, then

$$
\begin{aligned}
& e_{1, \mathrm{j}_{s}} \zeta_{1, \mathrm{j}_{s}} x+e_{1, \mathrm{j}_{s}}\left(1-\zeta_{1, \mathrm{j}_{s}}\right) y \in e_{1, \mathrm{j}_{s}} B \\
& e_{2, \mathrm{j}_{s}} \zeta_{2, \mathrm{j}_{s}} x+e_{2, \mathrm{j}_{s}}\left(1-\zeta_{2, \mathrm{j}_{s}}\right) y \in e_{2, \mathrm{j}_{s}} B
\end{aligned}
$$


Therefore,

$$
\begin{aligned}
\zeta x+(1-\zeta) y & =\zeta_{1, \mathrm{j}_{s}} e_{1, \mathrm{j}_{s}} x+\zeta_{2, \mathrm{j}_{s}} e_{2, \mathrm{j}_{s}} x+\left(1-\zeta_{1, \mathrm{j}_{s}}\right) e_{1, \mathrm{j}_{s}} y+\left(1-\zeta_{2, \mathrm{j}_{s}}\right) e_{2, \mathrm{j}_{s}} y \\
& =\zeta_{1, \mathrm{j}_{s}} e_{1, \mathrm{j}_{s}} x+\left(1-\zeta_{1, \mathrm{j}_{s}}\right) e_{1, \mathrm{j}_{s}} y+\zeta_{2, \mathrm{j}_{s}} e_{2, \mathrm{j}_{s}} x+\left(1-\zeta_{2, \mathrm{j}_{s}}\right) e_{2, \mathrm{j}_{s}} y
\end{aligned}
$$

and $[\zeta x+(1-\zeta) y] \in e_{1, \mathrm{j}_{s}} B+e_{2, \mathrm{j}_{s}} B$. This proves the assertion.

Especially, if we take $H_{2}$-modules $X=H_{2}$, three different convex set definitions which are meaningful geometrically are given for the first time in the following definition.

Definition 4.20. Let $B \subseteq M_{k} \subseteq H_{2}$ be a subset of $H_{2}$-module $H_{2}$ for $k=1,2,3$. For all $x, y \in B$ and all real numbers $\lambda \in \mathbb{R}$ such that $0 \leq \lambda \leq 1$, then

i) $B$ is called $S M_{k}-$ convex set if $\lambda x+(1-\lambda) y \in B$ and $y \in S M_{k}(x)$,

ii) $B$ is called $N M_{k}-$ convex set if $\lambda x+(1-\lambda) y \in B$ and $y \in N M_{k}(x)$,

iii) $B$ is called $T M_{k}-$ convex set if $\lambda x+(1-\lambda) y \in B$ and $y \in T M_{k}(x)$.

Theorem 4.21. Let $B \subseteq M_{k} \subseteq H_{2}$ be a $S M_{k}$-convex subset of $H_{2}$-module $H_{2}$. The sets $e_{1, j_{s}} B=e_{1, j_{s}} B_{1, j_{s}}$ and $e_{2, j_{s}} B=e_{2, j_{s}} B_{2, j_{s}}$ are, respectively $s, k=1,2,3$,

i) $S M_{k}-$ convex subsets of $\mathrm{H}_{2}-$ modules $e_{1, j_{s}} \mathrm{H}_{2}$ and $e_{2, j_{s}} \mathrm{H}_{2}$ if $s=k$,

ii) $N M_{k}-$ convex subsets of $H_{2}-$ modules $e_{1, j_{s}} H_{2}$ and $e_{2, j_{s}} H_{2}$ if $s \neq k$.

Proof. i) Let us take $t_{1}, t_{2} \in e_{1, \mathrm{j}_{s}} B_{1, \mathrm{j}_{s}}$ for $s=k s, k=1,2,3$. There are arbitrary elements $x, y \in B$ such that $t_{1}=e_{1, \mathrm{j}_{s}} x$ and $t_{2}=e_{1, \mathrm{j}_{s}} y$. Since the set $B$ is a $S M_{k}-$ convex set, $\lambda x+(1-\lambda) y \in B$ where $y \in S M_{k}(x)$ and $\lambda \in \mathbb{R}$ such as $0 \leq \lambda \leq 1$. Moreover, we find

$$
\begin{aligned}
e_{1, \mathrm{j}_{s}}(\lambda x+(1-\lambda) y) & =\lambda e_{1, \mathrm{j}_{\mathrm{s}}} x+(1-\lambda) e_{1, \mathrm{j}_{s} y} y \\
& =\lambda t_{1}+(1-\lambda) t_{2} \in e_{1, \mathrm{j}_{s}} B \\
& =e_{1, \mathrm{j}_{s}} B_{1, \mathrm{j}_{s}} .
\end{aligned}
$$

Also, if $t_{1}, t_{2} \in e_{1, \mathrm{j}_{s}} B_{1, \mathrm{j}_{s}}$, then $t_{1}=e_{1, \mathrm{j}_{s}} t_{1}$ and $t_{2}=e_{1, \mathrm{j}_{s}} t_{2}$. When $s=k$, if $y \in S M_{k}(x)$, then $t_{2} \in S M_{k}\left(t_{1}\right)$ from [14]. Consequently, the sets $e_{1, \mathrm{j}_{s}} B$ are $S M_{k}$-convex subsets of the $H_{2}$-modules $e_{1, \mathrm{j}_{s}} H_{2}$. Similarly, it is proven that the sets $e_{2, \mathrm{j}_{s}} B$ are $S M_{k}$-convex subsets of $H_{2}$-modules $e_{2, \mathrm{j}_{s}} H_{2}$ for $s=k$.

ii) Following a similar way to the first proof and considering that if $y \in S M_{k}(x)$, then $t_{2} \in N M_{k}\left(t_{1}\right)$ for $s \neq k$ from [14], it is proven that the sets $e_{1, \mathrm{j}_{s}} B$ are $N M_{k}-$ convex subsets of $H_{2}$-modules $e_{1, \mathrm{j}_{s}} H_{2}$. Similarly, the sets $e_{2, \mathrm{j}_{s}} B$ are $N M_{k}-$ convex subsets of $\mathrm{H}_{2}-$ modules $e_{2, \mathrm{j}_{s}} \mathrm{H}_{2}$, too.

Theorem 4.22. Let $B \subseteq M_{k} \subseteq H_{2}$ be a $N M_{k}$-convex subset of $H_{2}$-module $H_{2}$. The sets $e_{1, j_{s}} B=e_{1, j_{s}} B_{1, j_{s}}$ and $e_{2, j_{s}} B=e_{2, j_{s}} B_{2, j_{s}}$ are $\mathrm{NM}_{k}$-convex sets of $\mathrm{H}_{2}$-modules $e_{1, j_{s}} H_{2}$ and $e_{2, j_{s}} H_{2}$ respectively $s, k=1,2,3$ where $s=k$ or $s \neq k$.

Theorem 4.23. Let $B \subseteq M_{k} \subseteq H_{2}$ be a TM $M_{k}$-convex subset of $H_{2}$-module $H_{2}$. The sets $e_{1, j_{s}} B=e_{1, j_{s}} B_{1, j_{s}}$ and $e_{2, j_{s}} B=e_{2, j_{s}} B_{2, j_{s}}$ are, respectively $s, k=1,2,3$,

i) $T M_{k}$-convex subsets of $\mathrm{H}_{2}-$ modules $e_{1, j_{s}} \mathrm{H}_{2}$ and $e_{2, j_{s}} \mathrm{H}_{2}$ if $s=k$,

ii) $N M_{k}$-convex subsets of $H_{2}$-modules $e_{1, j_{s}} H_{2}$ and $e_{2, j_{s}} H_{2}$ if $s \neq k$.

Definition 4.24. Let $X$ be a $H_{2}$-module and $\varnothing \neq B \subseteq X$. Some real numbers $\lambda>0$ for all $x \in X$ and for all scalars $\mu \in M_{k} \subseteq H_{2}$ such that $|\mu|_{j_{k}} \geq \lambda(k=1,2,3)$,

i) $B$ is called $S M_{k}-$ absorbing set if $x \in \mu B$ and $\mu \in S M_{k}(O)$,

ii) $B$ is called $T M_{k}$-absorbing set if $x \in \mu B$ and $\mu \in T M_{k}(O)$.

Theorem 4.25. Let $X$ be a $H_{2}-$ module and $\varnothing \neq B \subseteq X$. If the subset $B$ is a SM $M_{k}$-absorbing set $(k=1,2,3)$. Then 
i) $e_{1, j_{s}} B=e_{1, j_{s}} B_{1, j_{s}}$ and $e_{2, j_{s}} B=e_{2, j_{s}} B_{2, j_{s}}$ are $S M_{k}$-absorbing sets of $H_{2}$-modules $e_{1, j_{s}} X=X_{1, j_{s}}$ and $e_{2, j_{s}} X=X_{2, j_{s}}$ for $s=k=1$, respectively.

ii) $e_{1, j_{s}} B=e_{1, j_{s}} B_{1, j_{s}}$ and $e_{2, j_{s}} B=e_{2, j_{s}} B_{2, j_{s}}$ are SH-absorbing sets of $H$-modules $e_{1, j_{s}} X=X_{1, j_{s}}$ and $e_{2, j_{s}} X=X_{2, j_{s}}$ for $s, k=2,3$ and $s=k$, respectively.

Proof. i) Let's take $\tilde{x} \in e_{1, \mathrm{j}_{1}} X$ for $s=1$. There is an element $x \in X$ such that $\tilde{x}=e_{1, \mathrm{j}_{1}} x$. Since $B$ is $S M_{1}-$ absorbing set for $k=1, x \in \mu B$ for some real numbers $\lambda>0$ and all scalars $\mu \in S M_{1}(O)$ such as $|\mu|_{\mathrm{j}_{1}} \geq \lambda$. If we take $\mu=\mu_{1, \mathrm{j}_{1}} e_{1, \mathrm{j}_{1}}+\mu_{2, \mathrm{j}_{1}} e_{2, \mathrm{j}_{1}}$, then

$$
\tilde{x}=e_{1, \mathrm{j}_{1}} x \in e_{1, \mathrm{j}_{1}} \mu B=e_{1, \mathrm{j}_{1}}\left(\mu_{1, \mathrm{j}_{1}} e_{1, \mathrm{j}_{1}}+\mu_{2, \mathrm{j}_{1}} e_{2, \mathrm{j}_{1}}\right) B=\mu_{1, \mathrm{j}_{1}} e_{1, \mathrm{j}_{1}} B
$$

is obtained. On the other hand, if $\mu \in S M_{1}(O)$, then $|\mu|_{\mathrm{j}_{1}}=\left|\mu_{1, \mathrm{j}_{1}}\right|_{\mathrm{j}_{1}}$ and hence $\mu_{1, \mathrm{j}_{1}} \in S M_{1}(O)$ from the [14]. Consequently, $\tilde{x} \in \mu_{1, \mathrm{j}_{1}} e_{1, \mathrm{j}_{1}} B$ for some real numbers $\lambda>0$ and for all scalars $\mu_{1, \mathrm{j}_{1}} \in S M_{1}(O)$ such that $\left|\mu_{1, \mathrm{j}_{1}}\right|_{\mathrm{j}_{1}}=|\mu|_{\mathrm{j}_{1}} \geq \lambda$. In that case, the set $e_{1, \mathrm{j}_{1}} B=e_{1, \mathrm{j}_{1}} B_{1, \mathrm{j}_{1}}$ is a $S M_{1}$-absorbing subset of $H_{2}$-module $e_{1, \mathrm{j}_{1}} X=e_{1, \mathrm{j}_{1}} X_{1, \mathrm{j}_{1}}$.

ii) Consider $\tilde{x} \in e_{1, \mathrm{j}_{2}} X$ for $s=k=2$ where $\tilde{x}=e_{1, \mathrm{j}_{2}} x$ and $x \in X$. Since $B$ is $S M_{2}$-absorbing set for $k=2, x \in \mu B$ for some real numbers $\lambda>0$ and for all scalars $\mu \in S M_{2}(O)$ such that $|\mu|_{\mathrm{j}_{2}} \geq \lambda$. Hence

$$
\tilde{x}=e_{1, \mathrm{j}_{2}} x \in e_{1, \mathrm{j}_{2}} \mu B=e_{1, \mathrm{j}_{2}}\left(\mu_{1, \mathrm{j}_{2}} e_{1, \mathrm{j}_{2}}+\mu_{2, \mathrm{j}_{2}} e_{2, \mathrm{j}_{2}}\right) B=\mu_{1, \mathrm{j}_{2}} e_{1, \mathrm{j}_{2}} B
$$

is obtained where $\mu=\mu_{1, \mathrm{j}_{2}} e_{1, \mathrm{j}_{2}}+\mu_{2, \mathrm{j}_{2}} e_{2, \mathrm{j}_{2}}$. On the other hand, $|\mu|_{\mathrm{j}_{2}}=\left|\mu_{1, \mathrm{j}_{2}}\right|_{H}$ and $\mu_{1, \mathrm{j}_{2}} \in S H(O)$ from the [14]. Hence, the set $e_{1, \mathrm{j}_{2}} B=e_{1, \mathrm{j}_{2}} B_{1, \mathrm{j}_{2}}$ is $S H$-absorbing set of $H_{2}$-modules $e_{1, \mathrm{j}_{2}} X=e_{1, \mathrm{j}_{2}} X_{1, \mathrm{j}_{2}}$. The case $s=k=3$ can be proved by using the similar way.

Theorem 4.26. Let $X$ be a $H_{2}-$ module and $\varnothing \neq B \subseteq X$. If the subset $B$ is $T M_{k}-$ absorbing set for $k=1,2,3$, then

i) $e_{1, j_{s}} B=e_{1, j_{s}} B_{1, j_{s}}$ and $e_{2, j_{s}} B=e_{2, j_{s}} B_{2, j_{s}}$ are $T M_{k}$-absorbing sets of $H_{2}$-modules $e_{1, j_{s}} X=X_{1, j_{s}}$ and $e_{2, j_{s}} X=X_{2, j_{s}}$ for $s=k=1$,

ii) $e_{1, j_{s}} B=e_{1, j_{s}} B_{1, j_{s}}$ and $e_{2, j_{s}} B=e_{2, j_{s}} B_{2, j_{s}}$ are $T H$-absorbing sets of $H$-modules $e_{1, j_{s}} X=X_{1, j_{s}}$ and $e_{2, j_{s}} X=X_{2, j_{s}}$ for $s, k=2,3$ and $s=k$.

Topological bihyperbolic module which is not previously found in the literature is defined as follows.

Definition 4.27. Let $X$ be a $H_{2}-$ module and $\tau$ is a Hausdorff topology on $X$. If the operations

$$
\begin{aligned}
& +: X \times X \rightarrow X \\
& \cdot: H_{2} \times X \rightarrow X
\end{aligned}
$$

are continuous, then the pair $(X, \tau)$ is called a topological bihyperbolic module or topological $\mathrm{H}_{2}-$ module.

When the topological vector spaces were introduced in [21], there was a condition such that the single point sets are closed according to the topology on it. The topological vector spaces are Hausdorff space with this condition. But, when the topological vector spaces were introduced in the literature, it was not said that the topology which is corresponding with the topological vector spaces are Hausdorff topology. The reason for this is usually that most of the spaces already provide the Hausdorff property. For instance, the topologies generated by norms on the normed vector space or the topologies generated by metrics are Hausdorff topologies. These structures which are using in the functional analysis frequently appear in the topological vector spaces, too. Although this article has more general structure than these structures, the topology corresponding with $\mathrm{H}_{2}$-module is taken as Hausdorff topology, unless otherwise stated.

Theorem 4.28. Let $(X, \tau)$ be a topological $\mathrm{H}_{2}$-module. The families

$$
\begin{aligned}
& \tau_{1, j_{s}}=\left\{e_{1, j_{s}} G: G \in \tau\right\}, \\
& \tau_{2, j_{s}}=\left\{e_{2, j_{s}} G: G \in \tau\right\}
\end{aligned}
$$

are Hausdorff topologies on the $H_{2}-$ modules $X_{1, j_{s}}$ and $X_{2, j_{s}}$ for $s=1,2,3$, respectively. Especially, they are Hausdorff topologies on $H$-modules $X_{1, j_{s}}$ and $X_{2, j_{s}}$ for $s=2,3$, respectively. 
Theorem 4.29. Let $(X, \tau)$ be a topological $H_{2}-$ module and $\left(X_{i, j_{s}}, \tau_{i, j_{s}}\right)$ be topological spaces for $s=1,2,3$ and $i=1,2$. Then, the operations

$$
\begin{array}{r}
+: X_{i, j_{s}} \times X_{i, j_{s}} \rightarrow X_{i, j_{s}}, \\
\cdot: H_{2} \times X_{i, j_{s}} \rightarrow X_{i, j_{s}}
\end{array}
$$

are continuous.

Especially, the subsets $X_{1, \mathrm{j}_{s}}$ and $X_{2, \mathrm{j}_{s}}$ are $H$-modules of the $H_{2}$-modules $X$, since $\zeta_{1, \mathrm{j}_{s}}, \zeta_{2, \mathrm{j}_{s}} \in H$ where $\zeta=\zeta_{1, \mathrm{j}_{s}} e_{1, \mathrm{j}_{s}}+\zeta_{2, \mathrm{j}_{s}} e_{2, \mathrm{j}_{s}} \in H_{2}$ for $s=2,3$. Hence, the operations

$$
\begin{gathered}
+: X_{i, \mathrm{j}_{s}} \times X_{i, \mathrm{j}_{s}} \rightarrow X_{i, \mathrm{j}_{s}} \\
\cdot: H \times X_{i, \mathrm{j}_{s}} \rightarrow X_{i, \mathrm{j}_{s}}
\end{gathered}
$$

are continuous for $s=2,3$ and $i=1,2$, too.

Corollary 4.30. Let $(X, \tau)$ be a topological $H_{2}$-module. The pair $\left(X_{i, j_{s}}, \tau_{i, j_{s}}\right)$ are topological $H_{2}-$ modules for $s=1,2,3$ and $i=1,2$. Especially, the pair $\left(X_{i, j_{s}}, \tau_{i, j_{s}}\right)$ are topological $H$-modules for $s=2,3$ and $i=1,2$, too.

Theorem 4.31. Let $(X, \tau)$ be a topological $H_{2}-$ module. If the operation $T_{y}: X \rightarrow X$ for any $y \in X$ is defined as $T_{y}(x)=x+y$ for all $x \in X$, then it is a homeomorphism.

Proof. The operation $T_{y}$ is continuous by the definition of the topological module and it is bijective by the axioms of the module. Moreover, $T_{y}^{-1}(x)=T_{-y}(x)=x-y$ and $T_{y} \circ T_{-y}=T_{-y} \circ T_{y}=I$ are obtained. Therefore, the operation $T_{y}^{-1}=T_{-y}$ is also continuous. Consequently, the operation $T_{y}$ is a homeomorphism.

Theorem 4.32. Let $(X, \tau)$ be a topological $H_{2}-$ module. If the operation $M_{\zeta}: X \rightarrow X$ for any $\zeta \in H_{2}^{*}$ is defined as $M_{\zeta}(x)=\zeta x$ for all $x \in X$, then it is a homeomorphism.

Proof. The operation $M_{\zeta}$ is continuous by the definition of the topological $H_{2}$-module and it is bijective by the axioms of the module. $M_{\zeta}^{-1}(x)=M_{1 / \zeta}(x)=\frac{x}{\zeta}$ for $\zeta \in H_{2}^{*}$ and $M_{\zeta} \circ M_{1 / \zeta}=M_{1 / \zeta} \circ M_{\zeta}=I$ are obtained. Hence, the operation $M_{\zeta}^{-1}=M_{1 / \zeta}$ is also continuous. This completes the proof.

We will investigate the properties of the interiors and the closures of the subsets of the $H_{2}$-module $X$ in the following theorems. $A^{\circ}$ represents the interior of the set $A$ and $\bar{A}$ represents the closure of the set $A$.

Theorem 4.33. Let $X$ be a topological $H_{2}$-module and $\varnothing \neq B \subseteq X$. Then the followings are satisfied.

i) $\left(e_{1, j_{s}} B\right)^{\circ}=e_{1, j_{s}} B^{\circ}$ and $\left(e_{2, j_{s}} B\right)^{\circ}=e_{2, j_{s}} B^{\circ}(s=1,2,3)$.

ii) $\overline{\left(e_{1, j_{s}} B\right)}=e_{1, j_{s}} \bar{B}$ and $\overline{\left(e_{2, j_{s}} B\right)}=e_{2, j_{s}} \bar{B}(s=1,2,3)$.

Proof. i) Let's take $x \in\left(e_{1, \mathrm{j}_{s}} B\right)^{\circ}$. There exists an open neighbourhood $G \subseteq X$ such that $x \in e_{1, \mathrm{j}_{s}} G \subseteq e_{1, \mathrm{j}_{s}} B$ where $x=e_{1, \mathrm{j}_{s}} y$ and $y \in G$. Clearly, $y \in G^{\circ}$. Thus, $x=e_{1, \mathrm{j}_{s}} y \in e_{1, \mathrm{j}_{s}} B^{\circ}$ and $\left(e_{1, \mathrm{j}_{s}} B\right)^{\circ} \subseteq e_{1, \mathrm{j}_{s}} B^{\circ}$ are obtained. Conversely, let's take $y \in B^{\circ}$. Hence, $e_{1, \mathrm{j}_{s}} y \in e_{1, \mathrm{j}_{s}} B^{\circ}$. If $y \in B^{\circ}$, then there is an open neighbourhood $G \subseteq X$ such as $y \in G \subseteq B$. Therefore, $e_{1, \mathrm{j}_{s}} y \in e_{1, \mathrm{j}_{s}} G \subseteq e_{1, \mathrm{j}_{s}} B$. Since $G$ is the open set in $X$, the set $e_{1, \mathrm{j}_{s}} G$ is also an open set in $e_{1, \mathrm{j}_{s}} X$ from Theorem 4.28, too. Consequently, $e_{1, \mathrm{j}_{s}} y \in\left(e_{1, \mathrm{j}_{s}} B\right)^{\circ}$ and $e_{1, \mathrm{j}_{s}} B^{\circ} \subseteq\left(e_{1, \mathrm{j}_{s}} B\right)^{\circ}$ are obtained. These two inclusions prove the assertion. Similarly, it can be shown that $\left(e_{\mathrm{j}_{s}}^{2} B\right)^{\circ}=e_{\mathrm{j}_{s}}^{2} B^{\circ}$.

ii) Let's take $x \in \overline{\left(e_{1, \mathrm{j}_{s}} B\right)}$. There exists a net $\left\{x_{l}\right\} \in e_{1, \mathrm{j}_{s}} B$ such that $\left\{x_{l}\right\} \rightarrow x$. Moreover, the net $\left\{y_{l}\right\} \in B$ where $\left\{x_{l}\right\}=\left\{e_{1, \mathrm{j}_{s}} y_{l}\right\}$ can be taken such as $\left\{y_{l}\right\} \rightarrow y$. Hence, $y \in \bar{B}$. This means that $\left\{x_{l}\right\}=\left\{e_{1, \mathrm{j}_{s}} y_{l}\right\} \rightarrow e_{1, \mathrm{j}_{s}} y$. Since the topological space $(X, \tau)$ is Hausdorff, the spaces $\left(e_{1, j_{s}} X, \tau_{1, j_{s}}\right)$ are Hausdorff, too. So, if there is the limit of a net in the subset $e_{1, \mathrm{j}_{s}} B \subseteq e_{1, \mathrm{j}_{\Omega}} X$, it is unique. Therefore, $x=e_{1, \mathrm{j}_{s}} y \in e_{1, \mathrm{j}_{s}} \bar{B}$. From here, the inclusion $\overline{\left(e_{1, \mathrm{j}_{s}} B\right)} \subseteq e_{1, \mathrm{j}_{s}} \bar{B}$ is obtained. Conversely, take $y \in \bar{B}$. Hence, $e_{1, \mathrm{j}_{s}} y \in e_{1, \mathrm{j}_{s}} \bar{B}$. If $y \in \bar{B}$, then there is a net $\left\{y_{l}\right\} \subseteq B$ such that $\left\{y_{l}\right\} \rightarrow y$. Therefore, there exists a net $\left\{e_{1, \mathrm{j}_{s}} y_{l}\right\} \subseteq e_{1, \mathrm{j}_{s}} B$ such as $\left\{e_{1, \mathrm{j}_{s}} y_{l}\right\} \rightarrow e_{1, \mathrm{j}_{s}} y$. So, $e_{1, \mathrm{j}_{s}} y \in \overline{\left(e_{1, \mathrm{j}_{s}} B\right)}$ and $e_{1, \mathrm{j}_{s}} \bar{B} \subseteq \overline{\left(e_{1, \mathrm{j}_{s}} B\right)}$ are obtained. Similarly, one can prove that $\overline{\left(e_{2, \mathrm{j}_{s}} B\right)}=e_{2, \mathrm{j}_{s}} \bar{B}$. 
Theorem 4.34. Let $X$ be a topological $H_{2}-$ module and $\varnothing \neq B \subseteq X$. If $B$ is a $H_{2}$-convex subset of $X$ then the following relations are satisfied for $s=1,2,3$.

i) $B^{\circ}=e_{1, j_{s}} B^{\circ}+e_{2, j_{s}} B^{\circ}$,

ii) $\bar{B}=e_{1, j_{s}} \bar{B}+e_{2, j_{s}} \bar{B}$,

iii) $B^{\circ}$ is $H_{2}-$ convex,

iv) $\bar{B}$ is $H_{2}-$ convex.

Proof. i) Take into consideration $x \in B^{\circ}$. Then $x=\left(e_{1, \mathrm{j}_{s}}+e_{2, \mathrm{j}_{s}}\right) x=e_{1, \mathrm{j}_{s}} x+e_{2, \mathrm{j}_{s}} x \in e_{1, \mathrm{j}_{s}} B^{\circ}+e_{2, \mathrm{j}_{s}} B^{\circ}$ since $e_{1, \mathrm{j}_{s}}+e_{2, \mathrm{j}_{s}}=1$. So $B^{\circ} \subseteq e_{1, \mathrm{j}_{s}} B^{\circ}+e_{2, \mathrm{j}_{s}} B^{\circ}$. On the other hand, since $B$ is $H_{2}$-convex, $B=e_{1, \mathrm{j}_{s}} B+e_{2, \mathrm{j}_{\mathrm{s}}} B$ from Theorem 4.18. Hence, $e_{1, \mathrm{j}_{s}} B^{\circ}+e_{2, \mathrm{j}_{s}} B^{\circ}$ is an open subset of the topological $H_{2}$-module $X$ where $e_{1, \mathrm{j}_{s}} B^{\circ}+e_{2, \mathrm{j}_{s}} B^{\circ} \subseteq e_{1, \mathrm{j}_{s}} B+e_{2, \mathrm{j}_{s}} B=B$. But, the largest open set contained in $B$ must be $B^{\circ}$. So, $e_{1, \mathrm{j}_{s}} B^{\circ}+e_{2, \mathrm{j}_{s}} B^{\circ} \subseteq B^{\circ}$. This completes the proof.

ii) If $x \in \bar{B}$ is taken, then $x \in e_{1, \mathrm{j}_{s}} \bar{B}+e_{2, \mathrm{j}_{s}} \bar{B}$ and $\bar{B} \subseteq e_{1, \mathrm{j}_{s}} \bar{B}+e_{2, \mathrm{j}_{s}} \bar{B}$ are obtained. Note that in a topological vector space $X$ if $A \subseteq X$ and $B \subseteq X$, then $\bar{A}+\bar{B} \subseteq \overline{A+B}$ [21]. Thus,

$$
e_{1, \mathrm{j}_{s}} \bar{B}+e_{2, \mathrm{j}_{s}} \bar{B}=\overline{e_{1, \mathrm{j}_{s}} B}+\overline{e_{2, \mathrm{j}_{s}} B} \subseteq \overline{e_{1, \mathrm{j}_{s}} B+e_{2, \mathrm{j}_{s}} B}=\bar{B}
$$

from Theorem 4.33.

iii) Since $B$ is $H_{2}-$ convex, $\zeta x+(1-\zeta) y \in B$ for all $x, y \in B$ and for all $\zeta \in H_{2}^{+}$such that $0 \leq \zeta \leq 1$. This means that $\zeta x+(1-\zeta) y$ is an element of $B$ when the elements $x$ and $y$ are scanning the set $B$. So, $\zeta B+(1-\zeta) B \subseteq B$ is obtained. $B^{\circ}=\zeta B^{\circ}+(1-\zeta) B^{\circ} \subseteq B$ since $B^{\circ} \subseteq B$. Assume that $\zeta=0$. Therefore, $\zeta B^{\circ}+(1-\zeta) B^{\circ}=B^{\circ} \subseteq B^{\circ}$. Now, let's take $\zeta \neq 0$. Since the addition and multiplication with scalar operations are homeomorphisms in $X$ and $B^{\circ}$ is an open set in $X, \zeta B^{\circ}+(1-\zeta) B^{\circ}$ is an open set, too. But, the largest open set contained in $B$ is $B^{\circ}$. So, $\zeta B^{\circ}+(1-\zeta) B^{\circ} \subseteq B^{\circ}$. Consequently, $B^{\circ}$ is a $H_{2}-$ convex set.

iv) Let $B$ be a $H_{2}-$ convex subset of the topological $H_{2}$-module $X$. Let's define an operation

$$
\begin{aligned}
\varphi_{\zeta}: X \times X & \rightarrow X \\
(x, y) & \rightarrow \zeta x+(1-\zeta) y
\end{aligned}
$$

for all $\zeta \in H_{2}^{+}$such that $0 \leq \zeta \leq 1$. Since $X$ is a topological $H_{2}$-module, the addition and the multiplication with scalar operations are continuous on $X$ and hence the operation $\varphi_{\zeta}$ is continuous, too. Moreover, since $B$ is $H_{2}-$ convex, $\varphi_{\zeta}(B \times B) \subseteq B$ for $\zeta \in H_{2}^{+}$such as $0 \leq \zeta \leq 1$. Therefore, $\overline{\varphi_{\zeta}(B \times B)} \subseteq \bar{B}$. So we get $\varphi_{\zeta} \overline{(B \times B)} \subseteq \overline{\varphi_{\zeta}(B \times B)}$ since the operation $\varphi_{\zeta}$ is continuous. Consequently, $\varphi_{\zeta}(\bar{B} \times \bar{B})=\varphi_{\zeta} \overline{(B \times B)} \subseteq \bar{B}$. Hence, $\bar{B}$ is a $H_{2}$-convex subset of the topological $H_{2}$-module $X$.

Theorem 4.35. Let $X$ be a topological $H_{2}-$ module and the subset $\varnothing \neq B \subseteq X$ be a $S M_{k}$-balanced subset of $X$ for $k=1,2,3$. Then, the sets $\bar{B}$ and $B^{\circ}$ are $S M_{k}-$ balanced sets under the condition $\theta \in B^{\circ}$ where $\theta$ is the unit element.

Proof. Let's take $\zeta \in S M_{k}(O)$ such that $\zeta \bar{\zeta}^{\mathrm{j}_{k}} \leq 1$. If $\zeta=0$, then $\zeta \bar{B}=\{\theta\} \subseteq \bar{B}$. We assume that $\zeta \neq 0$. Since $B \subseteq X$ is a $S M_{k}$-balanced subset, $\zeta B \subseteq B$. Hence $\overline{\zeta B} \subseteq \bar{B}$. Considering that the multiplication with the scalar operation is a homeomorphism for $\zeta \in H_{2}^{*}$ from Theorem 4.32, $\zeta \bar{B}=\overline{\zeta B} \subseteq \bar{B}$ is obtained. Therefore, $\bar{B}$ is a $S M_{k}$-balanced set. Assume that $\theta \in B^{\circ}$. First, if $\zeta=0$, then $\zeta B^{\circ}=\{\theta\} \subseteq B^{\circ}$. Secondly, let's take $\zeta \neq 0 . \zeta B \subseteq B$ since $B \subseteq X$ is a $S M_{k}$-balanced subset. Thus, $(\zeta B)^{\circ} \subseteq B^{\circ}$ and $\zeta B^{\circ}=(\zeta B)^{\circ} \subseteq B^{\circ}$ from Theorem 4.32. Consequently, $B^{\circ}$ is a $S M_{k}$-balanced set.

Theorem 4.36. Let $X$ be a topological $H_{2}$-module and the subset $\varnothing \neq B \subseteq X$ be a $N M_{k}$-balanced subset of $X$ for $k=1,2,3$. Then $\bar{B}$ is a $N M_{k}$-balanced set.

Proof. Let's take $\zeta \in N M_{k}(O)$ such that $\zeta \bar{\zeta}^{\mathrm{j}_{k}}=0$. If $\zeta=0$, then $\zeta \bar{B}=\{\theta\} \subseteq \bar{B}$. We assume that $\zeta \neq 0$. Since $B \subseteq X$ is a $N M_{k}$-balanced subset, $\zeta B \subseteq B$. Hence, $\overline{\zeta B} \subseteq \bar{B} . \zeta \bar{B} \subseteq \overline{\zeta B}$ from Theorem 4.32. Finally, $\zeta \bar{B} \subseteq \overline{\zeta B} \subseteq \bar{B}$ is obtained and so $\bar{B}$ is a $N M_{k}$-balanced set. 
The multiplication with scalar operation has inverse only for $\zeta \in H_{2}^{*}$. Since the inverse of the multiplication with scalar operation must be continuous so that $\zeta B^{\circ} \subseteq(\zeta B)^{\circ}, B^{\circ}$ do not have to be a $N M_{k}$-balanced set while the subset $B$ is a $N M_{k}$-balanced set.

Theorem 4.37. Let $X$ be a topological $H_{2}$-module and the subset $\varnothing \neq B \subseteq X$ be a $T M_{k}$-balanced subset of $X$ for $k=1,2,3$. Then, $\bar{B}$ and $B^{\circ}$ are $T M_{k}$-balanced sets under the condition $\theta \in B^{\circ}$ where $\theta$ is the unit element.

Theorem 4.38. Let $X$ be a topological $H_{2}-$ module. The followings are satisfied for $k=1,2,3$.

i) All neighbourhoods of the element $\theta$ contain a $S M_{k}-$ absorbing neighbourhood of the element $\theta$ in $X$.

ii) All neighbourhoods of the element $\theta$ contain a $S M_{k}$-balanced neighbourhood of the element $\theta$ in $X$.

iii) All $\mathrm{H}_{2}$-convex neighbourhoods of the element $\theta$ contain a $\mathrm{H}_{2}$-convex and $S M_{k}$-balanced neighbourhood of the element $\theta$ in $X$.

Proof. i) Let $U_{\theta}$ be any neighbourhood of $\theta \in X$ and $V_{x}$ be any neighbourhood of $x \in X$. If $\zeta=0$, then $M_{0}(x)=\theta$. Since the multiplication with the scalar operation $M_{\zeta}$ is continuous, $M_{A_{0}}\left(V_{x}\right) \subseteq U_{\theta}$. Also, there is a neighbourhood of radius $\lambda>0$ and center $0 \in H_{2}$ such as $A_{0} \subseteq M_{k} \subseteq H_{2}$. Therefore, there is a neighbourhood $W_{\theta} \subseteq U_{\theta}$ such that $\mu x \in W_{\theta}$, $|\mu|_{\mathrm{j}_{k}} \leq \lambda$ and $\mu \in\left(\operatorname{SM}_{k}(O) \cap A_{0}\right)$. Moreover, if we choose $\frac{1}{\lambda}=\delta$, then $\delta>0$ and $x \in \mu^{-1} W_{\theta}$ for the scalars $\mu$ such as $\left|\mu^{-1}\right|_{\mathrm{j}_{k}} \geq \delta$. Consequently, $W_{\theta}$ is a $S M_{k}$-absorbing subset of $X$.

ii) Let $U_{\theta}$ be any neighbourhood of the unit element $\theta \in X$. Since $M_{0}(\theta)=\theta$ and the multiplication with the scalar operation is continuous, there is a neighbourhood of $\theta$ such as $V_{\theta}$ and $\mu V_{\theta} \subseteq U_{\theta}$ where the elements of the neighbourhood of $0 \in H_{2}$ with radius $\delta>0$ are $\mu \in H_{2}$ and $|\mu|_{\mathrm{j}_{k}} \leq \delta$. Especially, let's choose $\mu \in S M_{k}(O)$. If we say $\underset{|\mu|_{j_{k}} \leq \delta}{\cup} \mu V_{\theta}=A_{\theta}$, then $\underset{|\mu|_{j_{k}} \leq \delta}{\cup} \mu V_{\theta}=\theta$ for $\mu=0$ and $\{\theta\} \subseteq U_{\theta}$. If $\mu \neq 0$, then $A_{\theta}$ is a neighbourhood of $\theta$ and $A_{\theta} \subseteq U_{\theta}$. Because the multiplication with the scalar operation is a homeomorphism only for the invertible scalars. On the other hand, take $x \in A_{\theta}$ and $\zeta \in S M_{k}(O)$ such that $|\zeta|_{\mathrm{j}_{k}} \leq 1$. Hence, there is some $y \in V_{\theta}$ such as $x=\mu y$. We get $\zeta x=\zeta \mu y \in A_{\theta}$ since $|\zeta \mu|_{\mathrm{j}_{k}}=|\zeta|_{\mathrm{j}_{k}}|\mu|_{\mathrm{j}_{k}} \leq \delta$. So, $A_{\theta}$ is a $S M_{k}$-balanced subset of the neighbourhood $U_{\theta}$.

iii) Let $U_{\theta} \subseteq X$ be a $H_{2}$-convex neighbourhood of $\theta \in X$ and $A=\underset{|\mu|_{j_{k}}=1}{\cap} \mu U_{\theta}$. There is a $S M_{k}$-balanced neighbourhood of $\theta$ such that $V_{\theta} \subseteq U_{\theta}$ from the previous proposition. Hence, $\mu^{-1} V_{\theta}=V_{\theta}$ for $\mu \in S M_{k}(O)$ such that $|\mu|_{\mathrm{j}_{k}}=1$ and $V_{\theta} \subseteq \mu U_{\theta}$. Moreover, $V_{\theta} \subseteq A$. It appears that $A$ is a neighbourhood of $\theta$ and $\theta \in A^{\circ} \subseteq U_{\theta}$. Now, let's see that the set $A^{\circ}$ is a $H_{2}$-convex and $S M_{k}$-balanced subset. Since the images and inverse images of convex sets under linear transformations are convex, the sets $\mu U_{\theta}$ are $H_{2}$-convex for $\mu \in S M_{k}(O)$ such that $|\mu|_{\mathrm{j}_{k}}=1$. Also, the intersection of the $H_{2}$-convex sets is $H_{2}$-convex. So, the set $A=\underset{|\mu|_{j_{k}}=1}{\cap} \mu U_{\theta}$ is $H_{2}$-convex, too. Hence, the set $A^{\circ}$ is $H_{2}-$ convex from Theorem 4.34 (iii). Finally, since $\mu U_{\theta}$ are $H_{2}$-convex sets containing the element $\theta, \zeta \mu U_{\theta} \subseteq \mu U_{\theta}$ for all $\zeta \in H_{2}^{+}$such that $0 \leq \zeta \leq 1$. On the other hand, $\zeta \lambda A=\underset{|\mu|_{j_{k}}=1}{\cap} \zeta \lambda \mu U_{\theta}=\underset{|\mu|_{j_{k}}=1}{\cap} \zeta \mu U_{\theta} \subseteq \underset{|\mu|_{j_{k}}=1}{\cap} \mu U_{\theta}=A$ for $\lambda \in S M_{k}(O)$ such that $|\lambda|_{\mathrm{j}_{k}}=1$. Hence, the set $A$ is $S M_{k}$-balanced. $A^{\circ}$ is $S M_{k}$-balanced according to Theorem 4.35 since $\theta \in A^{\circ}$.

Theorem 4.39. Let $X$ be a topological $\mathrm{H}_{2}$-module. Then the following properties are provided for $k=1,2,3$.

i) All neighbourhoods of the element $\theta$ contain a $T M_{k}-$ absorbing neighbourhood of the element $\theta$ in $X$.

ii) All neighbourhoods of the element $\theta$ contain a $T M_{k}$-balanced neighbourhood of the element $\theta$ in $X$.

iii) All $H_{2}$-convex neighbourhoods of the element $\theta$ contain a $H_{2}$-convex and $T_{k}$-balanced neighbourhood of the element $\theta$ in $X$.

Since the multiplication with the scalar operation is a homeomorphism only for the scalars which have a multiplicative inverse, the neighbourhood of $\theta \in X$ does not contain $N M_{k}$-balanced neighbourhood. Also, a $H_{2}-$ convex neighbourhood of the element $\theta \in X$ does not contain a $N M_{k}$-balanced neighbourhood of the element $\theta$. 


\section{Acknowledgements}

This paper is based on the PhD dissertation undertaken by the first author, under the supervision of the second author, at the Institute of Natural Sciences, Sakarya University in 2020. The authors would like to express their sincere thanks to the editor and the anonymous reviewers for their helpful comments and suggestions.

\section{Funding}

There is no funding for this work.

\section{Availability of data and materials}

Not applicable.

\section{Competing interests}

The authors declare that they have no competing interests.

\section{Author's contributions}

All authors contributed equally to the writing of this paper. All authors read and approved the final manuscript.

\section{References}

[1] A.A. Pogorui, R.M. Rodriguez-Dagnino, R.D. Rodrigue-Said, On the set of zeros of bihyperbolic polynomials, Complex Var. Elliptic Equ., 53 (2008), no. 7, 685-690.

[2] A. Grothendieck, Topological vector spaces, Gordon and Breach Science Publishers, New York, 1973.

[3] C. Segre, Le rappresentazioni reali delle forme complesse e gli enti iperalgebrici (The real representation of complex elements and hyperalgebraic entities), Math. Annalen, 40 (1892), no. 3, 413-467.

[4] D. Alfsmann, H.G. Gockler, Hypercomplex bark-scale filter bank design based on allpass-phase specifications, Conference paper: Signal processing conference (EUSIPCO), Proceedings of the 20th European, Bucharest, Romania, 2012.

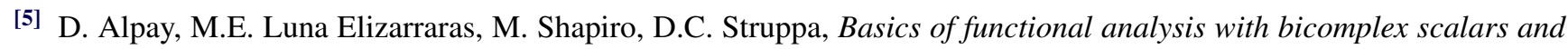
bicomplex Schur analysis, Springer Briefs in Mathematics, 2014.

[6] F. Catoni, D. Boccaletti, R. Cannata, ,V. Catoni, E. Nichelatti, P. Zampetti, The mathematics of Minkowski Space-Time with an introduction to commutative hypercomplex numbers, Birkhauser Verlag, Basel, Boston, Berlin, 2008.

[7] G. Baley Price, An introduction to multicomplex spaces and functions, Marcel Dekker Inc., New York, 1991.

[8] D. Bród, A. Szynal-Liana, I. Włoch, On the combinatorial properties of bihyperbolic balancing number, Tatra Mt. Math. Publ. 77 (2020), 27-38.

[9] D. Bród, A. Szynal-Liana, I. Włoch, On some combinatorial properties of bihyperbolic numbers of the Fibonacci type, Math. Methods Appl. Sci. Math. Methods Appl. Sci. 44(6) (2021), 4607-4615.

[10] J. Cockle, On certain functions resembling quaternions, and on a new imaginary in algebra, The London, Edinburgh, and Dublin Philosophical Magazine and Journal of Science, 33 (1848), no. 224. 435-439.

[11] J. Cockle, On a new imaginary in algebra, The London, Edinburgh, and Dublin Philosophical Magazine and Journal of Science, 34 (1849), no. 226. 37-47.

[12] J. Cockle, On the symbols of algebra and on the theory of Tessarines, The London, Edinburgh, and Dublin Philosophical Magazine and Journal of Science, 34 (1849), no. 231. 406-410.

[13] M. Bilgin, S. Ersoy, Algebraic properties of bihyperbolic numbers, Adv. Appl. Clifford Alg. 30 (2020), no. 13.

[14] S. Ersoy, M. Bilgin, Topolojik Bihiperbolik Modüller (Turkish) [Topological Bihyperbolic Modules], 31. National Mathematics Symposium, Erzincan Binali Yıldırım University, Erzincan, Turkey, 2018, pp. 69.

[15] M.E. Luna Elizarrarás, M. Shapiro, C.O. Perez-Regalado, On linear functionals and Hahn-Banach theorems for hyperbolic and bicomplex modules, Adv. Appl. Clifford Alg. 24 (2014), 1105-1129. 
[16] M.E. Luna Elizarrarás, M. Panza, M. Shapiro, D.C. Struppa, Geometry and Identity Theorems for Bicomplex Functions and Functions of a Hyperbolic Variable, Milan J. Math. 88 (2020), 247-261.

[17] R. Kumar, H. Saini, On Hahn Banach separation theorem for topological hyperbolic and topological bicomplex modules, arXiv preprint arXiv:1510.01538, 2015.

[18] R. Kumar, H. Saini, Topological bicomplex modules, Adv. Appl. Clifford Alg. 26 (2016), no. 4, 1249-1270.

[19] R. Larsen, Functional analysis, Marcel Dekker, New York, 1973.

[20] S. Olario, Complex numbers in n dimensions, North-Holland Mathematics Studies, Elsevier, vol. 190, 2002.

[21] W. Rudin, Functional analysis, 2nd Edition, McGraw Hill, New York, 1991. 